\title{
A SIMPLE RING SEPARATING CERTAIN RADICALS
}

\author{
by G. A. P. HEYMAN and W. G. LEAVITT \\ (Received 3 December, 1973; revised 7 May, 1974)
}

All rings considered will be associative. For a class $M$ of rings let $U M$ be the class of all rings having no non-zero homomorphic image in $M$. A hereditary class $M$ of prime rings is called a "special class" [see 1, p. 191] if it has the property that when $I \in M$ with $I$ an ideal of a ring $R$, then $R / I^{*} \in M$ where $I^{*}$ is the annihilator of $I$ in $R$, and the corresponding radical class $U M$ is then a "special radical". Let $S$ be the class of all subdirectly irreducible rings with simple heart.

Proposition 1 [1; Theorem 7, p. 202]. For any class $W$ of simple rings the class $M$ of all $R \in S$ with heart in $W$ is a special class so UM is a special radical.

For the class

$$
T=\{R \in S \text { whose heart contains an idempotent }\}
$$

the special radical $U T$ has been called the "Behrens radical" [see 3]. Notice that if $D$ is the class of all rings with unit, then the Brown-McCoy radical $U D \supseteq U T$. In attempting to characterize the Behrens radical one might consider the classes:

$$
\begin{aligned}
& V=\{R \in S \text { with von Neumann regular heart }\}, \\
& N=\{R \in S \text { whose heart contains a minimal left ideal }\} .
\end{aligned}
$$

Since both $V \subseteq T$ and $N \subseteq T$, we have

$$
U T \subseteq U D \cap U V \cap U N
$$

Note that since a semiprime ring contains a minimal left ideal if and only if it contains a minimal right ideal $[3$, p. 65$]$, we actually have

$$
N=\{R \in S \text { whose heart contains a minimal left and a minimal right ideal }\} .
$$

Using the following proposition, we will show that the inequality in (1) is proper.

PROPOSITION 2. The inclusion (1) is proper if and only if there exists a simple ring without unit, not von Neumann regular, and not containing a minimal one-sided ideal, but which does contain an idempotent.

Proof. The sufficiency is clear, so suppose that there exists some $R \in U D \cap U V \cap U N$ such that $R \notin U T$. Since radical classes are homomorphically closed, we may assume that $R \in T$. But $T$ is hereditary and since the radicals in (1) are special, they are also hereditary [1, Corollary 5, p. 195]. Thus the heart of $R$ is a simple ring in $T \cap U D \cap U V \cap U N$. 
The following two propositions are well-known, but we give the proofs for completeness:

PROPOSITION 3. If $I$ is a proper right ideal of a simple ring $R$ with unit, then $I / I \cap l(I)$ is a simple ring, where $l(I)$ is the left annihilator of I in $R$.

Proof. If $x \notin l(I)$ then $x I \neq 0$ so by simplicity $0 \neq R x I=R$. Also since $R$ has a unit, $I R=I$. Thus if $x \in I$ and $(x)$ is the ideal of $I$ generated by $x$, we have $I R x I=I R=I \subseteq(x)$. Thus $I=(x)$ and so $I / I \cap l(I)$ is simple.

PROPOSITION 4. No proper right ideal of a prime ring has a unit.

Proof. Let $0 \neq I$ be a right ideal of a prime ring $R$. If $e$ is the unit of $I$ then $R=I \oplus V$, where $V=\{x-e x\}$ for all $x \in R$. Since $e x=e x e$, we have $e x(y-e y)=0$ and so $I V=0$. But in a prime ring this implies that $V=0$, so that $I=R$ is not proper.

COROLLARY. If the left annihilator $l(I)=0$ for a proper right ideal $I$ of a simple ring $R$ with unit, then $I$ is a simple ring without unit.

Now consider the ring $R=Z_{2}[x, y, u, v]$ in non-commuting variables with relations $x u=y v=1, x v=y u=0$, and $u x=1+v y$. Note: $R$ can be regarded as a polynomial ring in which all elements are "reduced"; that is, no term contains $x u, y v, x v, y u$, or $u x$ and if such a term occurs in a product it is immediately reduced (using the above relations) [see 4 for details]. $R$ is a ring with unit which has been shown [5, Theorem 2, p. 307] to be simple.

We consider the right ideal $I=(u+1) R$.

LEMMA 1. I is a proper right ideal of $R$.

Proof. Certainly $I \neq 0$. Suppose that $I=R$. It will follow that $(u+1) a=1$ for some $a \in R$. But any longest term $w$ of $a$ will produce a longest term $u w$ (or $v y u^{\prime}$ if $w=x u^{\prime}$ ). In either case it will not be equal to any other term and so will not be cancelled in the product. Thus the product cannot equal 1 .

LEMMA 2. $l(I)=0$.

Proof. Suppose that $\left(h_{0}+h x+g y\right)(u+1)=0$, where $h_{0}$ is a function of $u, v$ alone. We obtain $h_{0}(u+1)+h+h x+g y=0$. A longest term $w$ of $h$ would produce an uncancelled longest term $w x$ and thus $h=0$. But then $g y=0$, so that $g=0$, and clearly $h_{0}(u+1)=0$ implies that $h_{0}=0$.

LEMMA 3. Iis not von Neumann regular.

Proof. If $(u+1)(u+1) a(u+1)=u+1$ for some $a \in R$, then, since $l(I)=0$, we have $(u+1)^{2} a=1$, contradicting the fact that $I$ is proper.

LEMMA 4. I does not contain a minimal right ideal. 
Proof. We show first that any non-zero right ideal $J$ of $I$ contains a non-zero element $(u+1) b$, where $b$ is a function of $u$ and $v$ alone. Suppose $0 \neq(u+1) a \in J$, where $a=h_{0}+$ $h_{1} x+h_{2} y$ with $h_{0}$ a function of $u$ and $v$ alone. Suppose first that $h_{1}=0$. Then if $h_{0} \neq 0$ we can multiply $(u+1) a$ on the right by $(u+1) u$, or if $h_{0}=0$ multiply by $(u+1) v$ and use induction on the length of $a$.

Thus suppose that $h_{1} \neq 0$. We use induction on the longest term of $a$ ending in $x$. We have $(u+1) a(u+1) u=(u+1)\left(h_{0} u^{2}+h_{0} u+h_{1} u+h_{1}\right)$. If any term ending in $x$ were to remain, it would be in $h_{1} u+h_{1}$ shorter than the longest such term in $h_{1} x$, and the result would follow by induction. If no term ending in $x$ remains but there is one ending in $y$, then again we can complete the proof by the argument of the first paragraph. We will thus have the desired result unless the product reduces to zero, that is unless $h_{0} u^{2}+h_{0} u+h_{1} u+h_{1}=0$. But then the terms of $h_{1}$ would end in $u$ (which is not permitted, since $h_{1} x$ is reduced).

It is now clear that $J$ cannot be minimal; for if $(u+1) b \in J$ with $b$ a function of $u$ and $v$ alone, then this would mean $(u+1) b I=J$. Thus $(u+1) b(u+1) a=(u+1) b$, for some $a \in R$, which would then also have to be a function of $u$ and $v$ alone, giving terms on the left that are too long.

\section{COROLlarY. I contains no minimal left ideal.}

THEOREM. The inclusion (1) is proper.

Proof. From Lemmas 1 and 2, the ring $I$ is simple without unit, and Lemmas 3 and 4 say that it is not von Neumann regular and does not contain a minimal one-sided ideal. However, $I$ does contain the idempotent $(u+1) v y$.

\section{REFERENCES}

1. V. A. Andrunakievitch, Radicals of Associative rings I, Mat. Sbornik 44 (1958), 179-212.

2. A. E. Behrens, Nichtassoziative Ringe, Math. Ann. 127 (1954), 441-452.

3. Nathan Jacobson, Structure of Rings, Amer. Math. Soc. Colloq. Publ. vol. 37, 1964.

4. W. G. Leavitt, The module type of a ring, Trans. Amer. Math. Soc. 103 (1962), 113-120.

5. W. G. Leavitt, The module type of homomorphic images, Duke Math. J. 32 (1965), 305-311.

University of the Orange Free State

UNIVERSITY OF NEBRASKA-LINCOLN, U.S.A. Bloemfontein, South Africa 